\title{
El uso de las redes sociales en el marco del Espacio Europeo de Educación Superior
}

\section{The use of social networks within the European Higher Education Area}

\author{
Ana I. Irimia-Diéguez (anairimia@us.es) \\ Filippo di Pietro (fdi@us.es) \\ Manuela Vega Pascual (mvegal@us.es) \\ Antonio Blanco Oliver (aj_blanco@us.es) \\ Universidad de Sevilla (España) \\ http://dx.doi.org/10.12795/EDUCADE.2014.i05.05
}

RESUMEN: Este trabajo recoge la experiencia de tres profesores noveles y una profesora mentora tras su participación en el proyecto "Formación del Profesorado Novel", organizado por el Instituto de Ciencias de la Educación (ICE) de la Universidad de Sevilla durante el curso académico 2011-2012. La incorporación de las redes sociales a la metodología docente universitaria, así como un uso intensivo de la plataforma virtual WebCT, se propusieron como dos de las actividades a desarrollar.

El objetivo del artículo es analizar si se produce una mejora en la participación activa y los resultados académicos del alumnado al incorporar tecnologías de la información y las comunicaciones (TICS) como base de las metodologías docentes. Para ello, se ha realizado un experimento de campo utilizando los resultados de tres asignaturas diferentes durante el curso académico 2011-2012: Introducción a las Finanzas, de primer curso, y Mercados Financieros Derivados, de tercer curso, ambas del grado en Finanzas y Contabilidad, y Dirección Financiera, de cuarto curso de la Licenciatura en Administración y Dirección de Empresas. Con este análisis buscamos: (a) contribuir a un mejor conocimiento a la hora de aplicar nuevas tecnologías como metodología docente; y (b) comprobar en qué medida una participación activa del alumno puede pronosticar una buena nota en el examen.

Tras la aplicación de estadística descriptiva y análisis de regresión logística (logit), nuestros resultados muestran que aquellos alumnos con una participación más activa en el seguimiento de la asignatura mejoran significativamente su rendimiento académico.

PALABRAS CLAVE: Redes sociales, Plataforma virtual, Innovación docente, Profesor novel, Profesor mentor.

ABSTRACT: This paper describes the experience of three novice lecturers and a mentor lecturer, after their participation in the project "Beginner Teacher Training" organized by the Institute of Education Sciences (ICE) at the University of Seville during the academic year 2011-2012. The incorporation of social networks to the university teaching methodology and an intensive use of the WebCT virtual platform were proposed as two of the activities to be developed.

Our objective in this article is to determine if there is an improvement in the student results when new technologies that require active student participation are incorporated as the basis of the teaching methodology. 
To this end, a field experiment is carried out by using the results of three different subjects for the academic course 2011-2012: Introduction to Finance (first year), Derivatives Financial Markets (third year), both belong to the degree in Finance and Accounting, and Corporate Finance (fourth year) of the degree in Business Administration and Management.

With this analysis we aim to: (a) contribute to a better understanding when implementing new technologies into the teaching methodology, and (b) verify to what extent a student's active participation can predict a good mark in the exam.

By using descriptive statistics and logistic regression analysis, the results show a marked improvement in the academic performance of those students whose participation is more active in the pursuit of the subject.

KEYWORDS: Networks, Virtual platform, Teaching innovation, Novel, Mentor.

\section{INTRODUCCIÓN}

La implantación del Espacio Europeo de Educación Superior (EEES) ha comportado un gran desafío para el profesorado universitario. Este marco fomenta la innovación en la metodología docente promoviendo una enseñanza más participativa con evaluación continua del alumnado, así como una mayor implicación por parte de los dos actores involucrados en este proceso: profesor y alumno. Además, la incorporación de nuevos grados ha supuesto un importante incremento de las necesidades docentes, generando la contratación de nuevo profesorado. Este hecho ha sido especialmente relevante en las Facultades de Ciencias Económicas y Empresariales, por un lado, y en la de Turismo y Finanzas, por otro, con la creación en esta última, del grado en Finanzas y Contabilidad el cual ha incrementado notablemente la actividad docente en el Departamento de Economía Financiera y Dirección de Operaciones. Dicha incorporación de nuevo profesorado, junto a la necesidad de cambios en las metodologías docentes surgidas bajo el contexto del EEES, generó la necesidad de replantear el binomio enseñanza-aprendizaje, enfocándolo hacia una relación más cercana con el alumnado, necesaria para establecer sistemas de evaluación continua. Adicionalmente, la utilización de las nuevas tecnologías se hace indispensable, no sólo por ser un factor cultural inherente a las nuevas generaciones, sino también por la elevada masificación que, de manera general, sufren las aulas.

Para mejorar las habilidades docentes y adaptarnos a la utilización de las TICs aplicadas a la docencia, un grupo de cuatro profesores decidimos participar en el Programa de Formación de Profesores Noveles organizado por el Instituto de Ciencias de la Educación (ICE) de la Universidad de Sevilla durante el curso académico 2011 2012. El grupo está formado por tres profesores noveles y una profesora mentora pertenecientes al departamento antes citado, todos ellos adscritos al área de conocimiento de Economía Financiera y Contabilidad.'

El motivo que nos llevó a participar en dicho curso fue ahondar en la mejora de la actividad docente mediante un profundo análisis de todos los aspectos docentes y a través del intercambio de experiencias y opiniones con otros profesores. Todos los miembros del grupo entendimos que era una gran oportunidad para reflexionar y

\footnotetext{
${ }^{1}$ Dos de los profesores noveles han sido becarios del Plan Propio de la Universidad de Sevilla; la tercera integrante novel es una profesora a tiempo parcial con experiencia profesional previa; $y$ la profesora mentora posee dieciséis años de experiencia en la Universidad.
} 
debatir sobre tareas relevantes de nuestro quehacer profesional y mejorar nuestra formación, adquiriendo nuevos recursos y conocimientos para desarrollar nuestra labor docente. Todo ello se traduciría en unos mejores resultados académicos y una mayor satisfacción personal y del alumnado. Además, la formación docente de los integrantes del grupo, salvo cursos muy específicos realizados a través del ICE, procedía básicamente de conversaciones informales con otros profesores del área, centradas en aspectos relacionados con la programación de las clases, contenido o material, y evaluación de los estudiantes. Asimismo, un uso más intensivo de las nuevas tecnologías nos parecía indispensable en el contexto del EEES.

El trabajo se estructura de la siguiente manera: a continuación, se definen los objetivos del trabajo; en el apartado siguiente se realiza una revisión de trabajos previos y se definen las hipótesis objeto de contraste; posteriormente, se explica la metodología seguida, para terminar con los resultados y conclusiones de la experiencia.

\section{OBJETIVOS}

Nuestra participación en el Programa de Formación de Profesores Noveles nos dio la oportunidad de introducir en el departamento un proyecto piloto de mejora de la enseñanza/aprendizaje mediante la introducción de herramientas tanto a nivel personal como tecnológico; en concreto, se produjo un uso más intensivo tanto de una de las redes sociales más ampliamente conocidas (Twitter) como de la plataforma virtual empleada en la Universidad de Sevilla (WebCT). La plataforma virtual WebCT está diseñada para ser utilizada como instrumento innovador de la docencia universitaria dentro del espacio EEES y es un punto virtual de contacto alumno/profesor/asignatura bajo la cual se pueden planificar, ejecutar y controlar las diversas tareas docentes. El objetivo planteado es comprobar si se produce una mejora en los resultados académicos del alumnado al incorporar como base de la metodología docente nuevas tecnologías que exigen una participación más activa del estudiante, utilizando los resultados de tres asignaturas diferentes del área de Finanzas durante el curso académico 2011-2012. Con ello buscamos: (a) contribuir a un mejor conocimiento a la hora de aplicar nuevas tecnologías como metodología docente; y (b) comprobar en qué medida una participación activa del alumnado puede pronosticar una buena nota en el examen.

El hecho de disponer de varias asignaturas y, en consecuencia, de un elevado número de grupos en los que impartíamos docencia, ha posibilitado la puesta en marcha de una serie de análisis comparativos entre el uso más o menos intensivo de la plataforma virtual o bien, del uso de la red social Twitter para socializar fuera de un contexto formal de enseñanza/aprendizaje.

\section{REVISIÓN DE LA LITERATURA Y FORMULACIÓN DE HIPÓTESIS}

La revisión de la literatura se realiza en dos bloques diferenciados aunque conectados. En primer lugar, señalamos aquellos artículos que, en la última década, estudian la relación entre metodologías de enseñanza activa y los resultados académicos, como recogen Miguel-Dávila et al. (2011). En segundo lugar, se incluyen aquellos estudios que se centran en el uso de las TICs y su impacto en el rendimiento del alumnado, por ser esta la forma más común de aplicar métodos de enseñanza activa.

Con respecto al primer bloque de revisión de la literatura, se observa que no hay resultados concluyentes sobre el uso de metodologías de enseñanza activa en los resultados académicos. En este sentido, diversos autores como Leeds et al. (1998) señalan que sus estudiantes de Economía obtenían mejores resultados con la lección magistral. Conclusiones similares muestran Catenazzi y Sommaruga (1999), con 
estudiantes de Informática o Collaud et al. (2000) con alumnos de Psicología. Finalmente, Sacristán-Díaz et al. (2012) muestran que no existe una mejora significativa en el rendimiento académico del alumnado cuando se implementan sistemas de enseñanza basados en actividades voluntarias y de trabajo autónomo del alumnado con respecto a los modelos de enseñanza tradicionales basados en la lección magistral, manual de referencia y examen final.

Sin embargo, otros trabajos han indicado que algunos métodos de enseñanza sí generan diferencias en el rendimiento del alumno. Oltra et al. (2011) muestran un mayor desarrollo de las competencias directamente relacionadas con el trabajo en equipo, especialmente cuando el proceso de enseñanza-aprendizaje se realiza de manera presencial. Alfalla-Luque et al. (2011), muestran ejemplos en los que se presenta una correlación positiva entre la realización de actividades obligatorias fuera de las aulas y el rendimiento del estudiante (Rayburn y Rayburn, 1999). Tang y Austin (2009) proponen un uso creativo de diferentes medios (proyector, transparencias en Power Point, vídeos, uso de internet y lección magistral) en aras de unos mayores niveles de aprendizaje, motivación, diversión y aplicación práctica de los conocimientos.

En relación al segundo bloque, Arbaugh et al. (2010) realizan una revisión del impacto de los sistemas de aprendizajes mixtos en varias disciplinas relacionadas con el área de organización empresarial entre los años 1994 a 2009. Yourstone et al. (2008) investigan si existen diferencias significativas en los resultados académicos entre el aprendizaje tradicional basado en la clase magistral y un aprendizaje basado en las TICs. Sus resultados concluyen que el hecho de obtener un feedback inmediato tiene un impacto positivo en el aprendizaje de los estudiantes. López-Pérez et al. (2011) analizan si el uso de las TICs mejora los resultados académicos, tomando una muestra de 17 grupos con un total de 1.431 estudiantes durante el año académico 2009/10. Sus resultados sugieren un efecto positivo del uso de las TICS porque reduce la tasa de absentismo y mejora los resultados académicos. De forma similar, Valentin et al. (2013) realizan un estudio sobre la relación existente el uso de las TICs, incluyendo una plataforma virtual, y los resultados académicos. Sus resultados también sugieren una relación positiva y una mejora de la satisfacción de los estudiantes. Finalmente, Wang (2008) propone un modelo teórico para facilitar la integración de las TICs en la enseñanza, basado en tres conceptos fundamentales: pedagogía, interacción social y tecnología.

Dentro de las TICs analizadas en los trabajos anteriores, el análisis de las redes sociales como metodología de enseñanza está menos estudiado por ser un instrumento de uso más reciente. Destacamos en esta línea el trabajo de Mateos-Aparicio (2012), que analiza las posibilidades de los sitios de redes sociales como entornos mediadores de enseñanza-aprendizaje. Otro estudio similar es el de Romero Frías (2010), que analiza el empleo de las wikis en la docencia universitaria. Los resultados obtenidos muestran tanto aspectos positivos (en relación con la difusión de conocimientos a la sociedad o la creación de un temario flexible, por ejemplo), como negativos (falta de participación activa de los alumnos como editores del wiki o escasas posibilidades de interacción social). En esta línea, Garandilla et al. (2011) analizan cómo la utilización de Herramientas Web 2.0, que posibilitan que los estudiantes interactúen, bases de datos y lectores de código de barras, permiten gestionar toda la información derivada del seguimiento diario del alumnado, y de la carga de trabajo distribuida entre clases magistrales, prácticas y ejercicios, tanto individuales como en grupo, y tanto dentro como fuera del aula.

Tras los objetivos planteados y la revisión de la literatura realizada, en este trabajo nos planteamos las siguientes hipótesis a contrastar: 
1. Hipótesis sobre la plataforma virtual:

La participación activa del alumnado, medida a través del uso intensivo de la plataforma virtual, mejora los resultados académicos.

2. Hipótesis relacionada con las redes sociales:

Las redes sociales como instrumento de evaluación continua mejoran los resultados académicos.

\section{METODOLOGÍA}

En este trabajo hemos realizado un experimento de campo para analizar los efectos que producen el uso de las redes sociales (Twitter) y de un uso intensivo de la plataforma virtual WebCT sobre la participación activa y el rendimiento académico del alumnado. Este estudio se ha realizado durante el curso 2011/2012 en tres asignaturas cuyos datos básicos se detallan seguidamente en la Tabla 1.

TABLA 1: CARACTERÍ́sticas de las ASIGNATURAS ANALIZADAS

\begin{tabular}{|c|c|c|c|c|c|}
\hline Asignatura & Titulación & Curso & $\begin{array}{l}\text { Número } \\
\text { de } \\
\text { Grupos }\end{array}$ & $\begin{array}{l}\text { Estudiantes } \\
\text { matriculados }\end{array}$ & $\begin{array}{c}\text { Estudiantes } \\
\text { presentad } \\
\text { os }\end{array}$ \\
\hline $\begin{array}{l}\text { Introducción a } \\
\text { las Finanzas }\end{array}$ & $\begin{array}{c}\text { Grado en Finanzas y } \\
\text { Contabilidad }\end{array}$ & Primero & 11 & 775 & 740 \\
\hline $\begin{array}{l}\text { Mercados } \\
\text { Financieros } \\
\text { Derivados }\end{array}$ & $\begin{array}{c}\text { Grado en Finanzas y } \\
\text { Contabilidad }\end{array}$ & Tercero & 5 & 210 & 184 \\
\hline $\begin{array}{l}\text { Dirección } \\
\text { Financiera }\end{array}$ & $\begin{array}{l}\text { Licenciatura en } \\
\text { Dirección y } \\
\text { Administración de } \\
\text { Empresas }\end{array}$ & Cuarto & 2 & 205 & 142 \\
\hline
\end{tabular}

La utilización de las redes sociales o la plataforma virtual creada ad hoc para la enseñanza activa más las clases magistrales nos dio la oportunidad de analizar cada herramienta, y comparar los resultados entre los diferentes grupos para evidenciar virtudes y defectos. Con este propósito, cada profesor decidió utilizar una tecnología concreta con el fin de intercambiar experiencias y poseer una visión global del efecto de la implantación de cada herramienta.

Así, uno de los profesores utilizó de forma intensiva WebCT (plataforma informática puesta a disposición del profesorado de la Universidad de Sevilla). El profesor que decidió utilizar esta herramienta había realizado un curso de 20 horas impartido por el Instituto de Ciencias de la Educación: "Aproximación a la Planificación y Diseño de la Docencia por Internet con WebCT", en el que había aprendido a manejar esta plataforma informática pudiendo así utilizar todas las herramientas que ofrece. No fue una herramienta más, si no que fue concebida como un lugar virtual alternativo indispensable. Por eso en esta asignatura se decidió ponderar con un $50 \%$ de la nota final la participación y rendimiento medido gracias al uso de la plataforma, a través de participar en foros, resolver pruebas y hacer exámenes.

Por otro lado, una de las profesoras se ofreció a utilizar las redes sociales. En un primer momento se ponderó cuál de las muchas redes sociales podía ser más apta para la 
enseñanza y de qué forma; después de evaluar las diferentes características de cada red social, la elección fue usar Twitter como herramienta de trabajo. Impartiendo clases en dos grupos de la misma asignatura, salió la ulterior idea de utilizar Twitter solo en uno de los dos grupos y seguir trabajando de forma "clásica" en el otro para tener otro término de comparación.

A modo de resumen, queremos señalar que en las tres asignaturas del experimento se utilizan contenido y sistemas de evaluación comunes para todos los grupos de una asignatura. Así, aunque hemos utilizado la plataforma WebCT en las tres asignaturas, en la asignatura de primer curso el uso se ha limitado a incluir el material a utilizar en clase, transparencias con contenidos teóricos, casos prácticos, lecturas, publicación de notas y uso del correo electrónico. En la asignatura de tercer curso se ha hecho un uso intensivo de la plataforma virtual, incluyendo además la evaluación de actividades y un control del seguimiento realizado por el alumnado. En la asignatura de cuarto curso también se ha utilizado WebCT y, adicionalmente, hemos experimentado el uso de la red social Twitter en uno de los grupos.

El análisis y comparación realizado se basa en nuestra experiencia bajo el marco del curso de innovación docente realizado. Por medio de estadísticas descriptivas y análisis logit hemos obtenido resultados que nos han posibilitado analizar el uso de la plataforma virtual y las redes sociales de forma cuantitativa y cualitativa.

\section{DESCRIPCIÓN DEL PROYECTO DE PROFESORES NOVELES}

El programa, que se ha desarrollado a lo largo de todo el curso académico 2011/12, está recogido en Sánchez Moreno (2008). Dicho programa es diferente para los profesores noveles y para la profesora mentora. En el caso de los profesores noveles, el proyecto consiste en el desarrollo de diversas tareas sobre diferentes aspectos de la educación universitaria (metodologías activas para la enseñanza universitaria, evaluación de alumnos y análisis de programas docentes, etc.). Además, se han trabajado mediante talleres presenciales tres módulos temáticos (logopedia y cuidado de la voz, control de la clase mediante lenguaje no verbal y relajación y expresión corporal).

El objetivo de estos módulos y talleres es que el profesor novel adquiera una formación integral en diversas materias que serán utilizadas diariamente en su actividad laboral (Sánchez Moreno y Mayor Ruiz, 2006). Además, se pretende que los profesores noveles conozcan las nuevas herramientas y formas de aprendizaje que gracias a las nuevas tecnologías han aparecido en los últimos años. Especialmente importante es el cambio producido en la forma de impartir las clases, partiendo de un tipo de clase basada en la lección magistral, la tendencia se encamina a otro tipo de clases sustentada en la interacción con los alumnos mediante herramientas informáticas y/o directamente mediante participación de los mismos en las clases a través de preguntas-respuestas.

En el caso de la profesora mentora, participó en diversas reuniones con otros profesores mentores, moderadas por expertos de la Facultad de Ciencias de la Educación. En dichos encuentros se intercambiaron experiencias entre profesores, mostrando las diferentes perspectivas y opiniones que surgen en el ámbito universitario como consecuencia tanto de las diferencias en la masificación de las aulas entre las distintas facultades de la Universidad de Sevilla como de las peculiaridades de las materias a impartir.

La dinámica de trabajo seguida por los profesores noveles fue el análisis y estudio colectivamente (es decir, los tres profesores noveles) de cada módulo del programa, entregando un informe final sobre diversas cuestiones planteadas por la dirección del 
programa. Como apoyo, los miembros del equipo contaban con bibliografía de referencia y un foro de debate en el que cada partícipe opinaba sobre los temas tratados, resolvían dudas y compartían experiencias. Además, se planificaron grabaciones de algunas clases impartidas por los profesores noveles. El visionado de las mismas fue realizado por todos los integrantes del grupo. En primer lugar, no se activó el sonido para focalizar en el lenguaje no verbal, y posteriormente, se visualizó con el sonido activado. El análisis del vídeo se realizó siguiendo el esquema de Mayor (1997), que sistematiza los aspectos a analizar en tres categorías: aspectos didácticos, visuales y orales.

Los principales problemas que encontraron los profesores noveles en la aplicación práctica de los contenidos teóricos tratados en el programa están relacionados con la gestión de la clase y las relaciones personales profesor-alumno. Las principales inquietudes que surgieron fueron:

a. ¿Cómo fomentar la participación en clase?

b. ¿Cómo gestionar al "listillo" de la clase?

C. ¿Cómo tratar a los alumnos conflictivos y habladores? Máxime en grupos numerosos.

d. ¿Cómo motivar a un grupo apático?

e. ¿Cómo realizar un uso más intensivo de la plataforma virtual?

f. ¿̇Es posible incorporar las redes sociales como una alternativa de comunicación alumno-profesor?

La última actividad del programa fue una reunión de los cuatro miembros del equipo con todos los equipos participantes en el programa. En dichas reuniones se compartieron la experiencia de los distintos grupos y se realizó una encuesta valorando los diversos aspectos del programa en aras de mejorarlo en próximas ediciones.

\section{EL USO DE LAS REDES SOCIALES}

Prácticamente la totalidad del alumnado tiene al menos un perfil en alguna red social, son usuarios de smartphones y de aplicaciones de comunicación como whatsapp, están familiarizados con el uso de las TICs y se les insta a usarlas para que se comuniquen con el profesorado, a través de mails o plataformas virtuales de docencia, e incluso para preparar trabajos y tareas en las asignaturas. En definitiva, conocen y usan las nuevas tecnologías, forman parte de su rutina diaria, y además les divierte. Por ello, nos pareció adecuado utilizar una red social para conectarnos y comunicarnos de forma complementaria a las clases y a los canales formales, mails y tutorías, que se mantuvieron.

Aunque nuestro objetivo era mejorar los resultados académicos del alumnado, al empezar a preparar la metodología para usar esta herramienta con los alumnos, se propuso conseguir que los alumnos hagan un uso adecuado de las redes sociales. Este fue el objetivo expuesto a los alumnos para conectarnos a través de Twitter, aunque realmente el principal objetivo de esta experiencia docente era mejorar los resultados académicos del grupo.

A la hora de seleccionar el medio de comunicación, se consideró que las plataformas virtuales que ofrece la Universidad de Sevilla, aunque cuentan con aplicaciones móviles, tienen diversas desventajas con respecto a Twitter, Facebook o Tuenti. Los inconvenientes más importantes son: (a) no tienen la misma operatividad y facilidad de uso; (b) los alumnos no la utilizan de forma tan intensiva ya que la plataforma de la 
Universidad de Sevilla tiene un carácter formal para los alumnos porque están directamente relacionadas con las asignaturas y los profesores; y (c) tiene mucho menor impacto ya que tienen menos usuarios al estar restringido exclusivamente a miembros pertenecientes a dicha institución. Por el contrario, el alumno utiliza las redes sociales comerciales (Twitter, Facebook o Tuenti), estando conectado a ellas casi de manera continua a través de sus aplicaciones móviles. En las redes sociales interactúan con personas afines o sobre aquellos aspectos que les interesan o les gustan, de manera que introducir la asignatura en ese medio en el que ellos se sienten cómodos y se divierten, al menos a priori, nos parecía adecuado para conectar con el alumnado.

La opción de Tuenti y Facebook fueron descartadas por tener un perfil de edad inferior y superior, respectivamente, a la media de edad del alumnado universitario (entre 18 y 23 años de edad en la mayoría de los casos). En cambio, Twitter se plantea como la opción más idónea. Es una red creada, al menos originariamente, con un marcado carácter profesional ya que trata de que sus usuarios compartan intereses comunes y no de interactuar con nuestros amigos. Además, Twitter nos permite estar conectados continuamente porque es una aplicación móvil y podemos activar alertas cuando recibamos Tuits, lo que nos avisa de que alguien se comunica con nosotros. Al ser una red abierta y conectada con el resto del mundo online, facilita enlazar rápida y fácilmente diferentes contenidos y nos permite conectar la materia de la asignatura con noticias o ejemplos de situaciones reales; o simplemente compartir aquello que se considera relevante.

Otra ventaja de Twitter es que no es necesario seguirnos todos los participantes, alumnos y profesor, para estar informados de nuestro interés común, ya que sólo se ha de seguir un hashtag, por lo que respeta la privacidad, antes comentada, para que todos nos sintamos libres a la hora de tuitear $y$, principalmente, el alumno no se sienta coartado a la hora de expresarse. Además, la mayoría de alumnos no tenían perfil en Twitter o no lo usaban, por lo que no había prejuicios a la hora de aceptar interactuar con el profesor.

La experiencia docente sobre la aplicación de Twitter comenzó con una charla informativa sobre la importancia que estaban adquiriendo las redes sociales como canales de búsqueda de empleo, y el cambio que se estaba originando, siendo las empresas las que buscaban a los profesionales que querían contratar a través de las mismas (Alcántara, 2012). Por ello, era muy importante controlar las fotos y toda la información que se comparte en las redes. Muchos alumnos desconocían este aspecto, por lo que se les instó a compartir información relativa a búsqueda de empleo, conocer las diferentes opciones de tener nuestro currículum online, crear una web personal, etc. Todo ello dirigido a convertirlos en candidatos visibles para las empresas, siendo activos en la búsqueda de empleo a través de las redes sociales.

Twitter comenzó a ser un elemento más en nuestras clases; usamos este canal para compartir noticias e información relacionada con la asignatura, y también para marcar el ritmo de las clases, contenido y trabajo previo a realizar, así como actividades propuestas. Por supuesto, también el alumno podía proponer ideas y compartir lo que considerara oportuno.

El aprendizaje de uso de esta red social fue colectivo pues la mayoría no estaba familiarizada con su uso. La primera actividad fue que todos los alumnos se abrieran una cuenta, crearan su perfil y empezaran a seguir a tuiteros de su interés; entre ellos, debían elegir alguna persona o perfil de empresa relacionado con la asignatura.

En concreto, esta herramienta se utilizó de diferentes maneras: 
- Herramienta de trabajo. Debido a la longitud y exceso de cálculos de los ejercicios de las asignaturas tratadas, se les solía hacer una pregunta muy concreta sobre el mismo, la cual sólo podían contestar si previamente habían trabajado el caso. Los Tuits, que sólo permiten un mensaje de 140 caracteres, lejos de resultar una limitación era una fuente de originalidad, en ocasiones hacían fotos a los folios donde habían desarrollado el caso y la enlazaban en el Tuit.

- Herramienta de investigación. Se les encargaba alguna actividad, que podía consistir en buscar el significado de un concepto, alguna noticia actual de prensa $\circ$ acontecimiento pasado que estuviese relacionado con la materia que se explicaba en clase en ese momento. El alumno debía realizar esa tarea de búsqueda y publicar su trabajo con un Tuit e incluir el enlace dando respuesta a lo que se le planteaba; y también leer los Tuits de los compañeros. En clase se comentaba aquello que se consideraba más relevante, siempre haciendo partícipes a los alumnos.

- Herramienta de comunicación. Se les recordaba qué casos y parte del temario tenían que trabajar y se les pedía una lectura previa para mejorar el ritmo de las clases. También se les pedían que propusieran ideas para el desarrollo de las clases.

- Herramienta de motivación. Complementariamente a todo el trabajo y comunicación relativa a la asignatura también se compartía con ellos diverso material que les sirviese de motivación, inspiración y ánimo. Podía compartir desde un spot publicitario, a un post, un vídeo de algún conferenciante, una canción o una reflexión.

El profesorado jugaba un papel de moderador, indicando si estaban acertados o no en la resolución de las actividades y animando a participar a aquellos que eran menos activos. Todo ello se hacía a través de un hashtag cuyo nombre fue decidido por el alumnado: \#ideasfinanzas.

La posibilidad de que el alumno no se animara a participar en esta actividad porque no quisiera compartir con su profesor, o con otros alumnos, alguna información suya personal, se solucionaba con el uso del hashtag. ${ }^{2}$ Paralelamente, cada alumno tenía la opción personal de decidir a quién seguir.

Esta metodología se les propuso de forma opcional, tenían libertad de decidir o no su participación, todo lo que se compartiera con esta herramienta era complementario al trabajo de clase. Los alumnos recibieron de buen grado la propuesta y participó un $91 \%$ de los alumnos que asistían a clase.

Twitter es una red social abierta y, por tanto, pública, y con una gran viralidad; en consecuencia, otras personas se comunicaban o eran seguidoras del hashtag, como por ejemplo los alumnos de otros grupos de la misma asignatura, incluidos alumnos del otro grupo, el de horario de mañana, los cuales se mostraban atraídos por la iniciativa.

\footnotetext{
2 En Twitter para comunicarnos debemos "seguirnos". En esta red, no tenemos amigos, sino que tenemos "seguidores", y decidimos a quien seguir. De manera que todo lo que un usuario tuitea, o escribe, lo leen sus seguidores. A su vez, un usuario lee lo que escriben las personas que sigue. Aunque también un usuario puede leer lo que escribe cualquier otro usuario sobre un tema de conversación determinado, a través de los hashtags, que son etiquetas de temas de conversación.
} 


\section{RESULTADOS}

\subsection{ANÁLISIS DE ASPECTOS DOCENTES}

Los aspectos de la docencia analizados, que siguiendo la bibliografía del proyecto, Ruiz (1992), Sarramona (1988) y Tapia (1991), se pueden clasificar en:

$\checkmark$ Aspectos didácticos: tipos de preguntas a realizar para motivar, para reflexionar, para obtener feedback, para mantener la atención, etc. Uso adecuado de los recursos comunes de la materia a impartir: transparencias, casos prácticos resueltos, propuestos, uso de manual, de bibliografía adicional, de Internet.

$\checkmark$ Aspectos visuales: expresión corporal, facial, la proximidad con el alumno y el movimiento en el aula entre otros.

$\checkmark$ Aspectos orales: evitar muletillas, manejar las pausas o silencios, el uso de la palabra y la voz, la vocalización, el ritmo del discurso, la claridad en la exposición.

\subsection{ANÁLISIS DE ASPECTOS TECNOLÓGICOS}

Especial atención dedicamos al análisis de los resultados obtenidos del empleo de las herramientas informáticas que, como hemos expuesto previamente, han sido empleadas en tres asignaturas: Introducción a las Finanzas, Mercados Financieros Derivados y Dirección Financiera.

En la asignatura Introducción a las Finanzas la utilización de WebCT ha sido marginal; por esta razón y por la falta de un seguimiento diferenciado de los alumnos que utilizaron esta herramienta y los que no, se hace difícil un análisis exhaustivo. Por ello, las conclusiones sobre esta asignatura se basan en la realización de actividades y la nota final, profundizando en las dos asignaturas donde sí hubo una utilización completa durante todo el curso de las dos herramientas presentadas anteriormente: WebCT para la asignatura de Mercados Financieros Derivados y Twitter para la asignatura de Dirección Financiera.

Como ha sido comentado con anterioridad, en la primera asignatura, Introducción a las Finanzas, la finalidad fue verificar si un mayor nivel de utilización de WebCT, medido a partir de los resultados de las evaluaciones continuas, implicaba una mejor preparación de la asignatura. Por ello, se utilizó una regresión logística ordinal -ordered logit- (Miguel-Dávila et al., 201 1; Krull y MacKinnon, 2001; Preacher et al., 2007). Como variable dependiente se utilizó la nota del examen valorada entre 0 y 10 , medida en los siguientes rangos $0-2,9 ; 3-4,9 ; 5-6,9 ;$ y $7-10$. Como variable independiente, se usó la media de las notas obtenidas en las tres pruebas de evaluación continua efectuadas durante el curso en una escala continua de 0 a 2.

La ecuación del modelo es la siguiente:

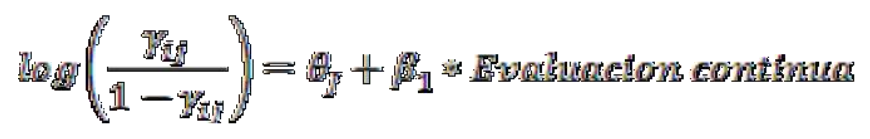

donde,

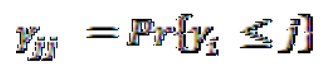

La Tabla 2, muestra una relación significativa entre el resultado de las evaluaciones continuas efectuadas por medio de WebCT y el resultado obtenido en el examen final. 
TABLA 2: Regresión logística entre el uso de la plataforma WebCT Y LA NOTA FINAL EN INTRODUCCIÓN A LAS FINANZAS

\begin{tabular}{|c|c|c|c|c|}
\hline \multicolumn{2}{|c|}{ Regresión logística ordinal } & \multicolumn{3}{|c|}{ número de observaciones = 740} \\
\hline \multicolumn{5}{|c|}{ log likelihood=-462,98429 } \\
\hline \multicolumn{5}{|l|}{ LR chi2=99,07 } \\
\hline \multicolumn{5}{|l|}{ Prob $>$ chi $2=0,00$} \\
\hline \multicolumn{5}{|l|}{ PseudoR2=0,1106 } \\
\hline Nota examen & coef. & Err. Estad. & Z & $P>z$ \\
\hline Notas_evaluaciones & 1,90 & 0,204 & 9,27 & $<, 0001^{* * *}$ \\
\hline
\end{tabular}

En la segunda asignatura, Mercados Financieros Derivados, una vez realizado el examen, nos pareció interesante analizar si los alumnos que habían participado más activamente en el uso de WebCT obtuvieron mejores notas que los que no emplearon esta herramienta intensivamente. Por ello, utilizamos una tabla clasificando los alumnos según la nota puesta por los profesores en función de la participación del alumno en las varias tareas de WebCT con su correspondiente nota media. La Tabla 3 muestra cómo los alumnos que participaron más activamente con WebCT obtuvieron una nota media más alta. En este sentido, hay que aclarar que la nota del examen final no corresponde con las notas finales que se han puesto en las actas, en cuanto que la nota del examen final no incluye la evaluación realizada a lo largo del curso.

TABLA 3: ESTADÍSTICOS DESCRIPTIVOS DEL USO DE LA PLATAFORMA WebCt en Mercados Financieros Derivados

\begin{tabular}{|c|c|c|}
\hline Utilización WebCT* & Promedio de nota final & Desv. Típica \\
\hline 0 & 3,38 & 1,53 \\
\hline 1 & 4,61 & 2,09 \\
\hline 2 & 4,23 & 1,78 \\
\hline 3 & 5,0 & 1,90 \\
\hline 4 & 5,38 & 1,94 \\
\hline 5 & 6,57 & 1,08 \\
\hline
\end{tabular}

* Nivel mínimo: 0, nivel máximo 5.

Con el objetivo de medir el esfuerzo de los alumnos en participar en WebCT, decidimos cuantificar el efecto de ese esfuerzo. Por ello, utilizamos, al igual que con la asignatura anterior, una regresión de tipo ordered logit. Como variable dependiente utilizamos la nota del examen valorada entre 0 y 10 , medida en rangos $0-2,9 ; 3-4,9 ; 5-6,9$; y $7-10$. Las variables dependientes son: grado de participación en la web y media ponderadas de las evaluaciones realizada por la web con escala de 0 a 2,5.

La ecuación del modelo es la siguiente: 


$$
\log \frac{Y_{i j}}{1 Y_{i j}}=\theta+\beta_{1} * \text { Participación WebCT }+\beta_{2} * \text { Notas evaluaciones }
$$

La Tabla 4 muestra que las relaciones de ambas variables independientes son significativas con respecto a la variable dependiente. La variable participación en WebCT es significativa con un nivel de significación inferior al 10\%. Es decir, hay menos de un $10 \%$ de probabilidad de que no sea estadísticamente significativa. La variable Notas Evaluaciones es significativa con un nivel de significatividad inferior al $1 \%$, esto es, existe menos de un $1 \%$ de probabilidad de que no sea significativa. En nuestro caso, hay una clara relación entre la participación activa en WebCT durante el curso y el resultado conseguido en el examen. Esta relación ya estaba manifestada en las Tablas 1 y 3 . Esto significa que fomentar una participación activa por medio de WebCT y el esfuerzo hecho por los alumnos de utilizar de forma intensiva esta herramienta ha dado sus frutos. Por otro lado, la Tabla 4 nos muestra que los alumnos que sacaron notas mejores en las evaluaciones hechas por WebCT también tienen más probabilidad de sacar mejor nota en el examen final.

TABLA 4: Regresión logística entre el uso de la plataforma WebCT, LAS eVAluACIONeS CONTINUAS Y LA NOTA FINAL en MERCAdos FinanCIEROS DeRIVAdos

\begin{tabular}{|l|c|c|c|c|}
\hline Regresión logística ordinal & \multicolumn{5}{l|}{ número de observaciones $=184$} \\
\hline $\begin{array}{l}\text { log likelihood=-235,7893 } \\
\text { LR chi2 }=13,7274 \\
\text { Prob>chi2=0,0328 } \\
\text { PseudoR2=0,3056 }\end{array}$ & coef. & err. estad. & z & P>z \\
\hline Nota examen & 0,335 & 0,1788 & 3,234 & $0,0706^{*}$ \\
\hline Participación WebCT & 2,59 & 0,4579 & 31,23 & $<, 0001^{* * *}$ \\
\hline Notas_evaluaciones & & &
\end{tabular}

Con respecto a la tercera asignatura, Dirección Financiera, el uso de Twitter como herramienta docente sólo se aplica en uno de los grupos, y es el que obtuvo peores resultados en la evaluación del primer parcial, como se puede observar en la Tabla 5. Este grupo constaba de 120 alumnos según el listado oficial, de los que asistían a clase regularmente 46 alumnos. El $91 \%$ de ellos, 41 alumnos, participan en la experiencia; de los cuales 37 superan la asignatura en la primera convocatoria final del curso. De esos 4 alumnos que participan en la experiencia y que no superan la asignatura, ninguno de ellos se presentó en esa convocatoria.

Los datos que presentamos recogen información hasta esta fecha, pero dos de ellos superaron la asignatura en convocatorias posteriores, uno en septiembre y otro en diciembre. Los otros dos alumnos, tampoco se presentaron a estas posteriores convocatorias. 
Ana I. Irimia, Filippo di Pietro, Manuela Vega, Antonio Blanco

El uso de las redes sociales en el maro del EEES

TAbla 5: Resultados del uso de Twitter en Dirección Financiera

\begin{tabular}{|c|c|c|c|c|}
\hline GRUPO con Twitter & \multicolumn{2}{|c|}{ PRIMER PARCIAL } & \multicolumn{2}{|c|}{ SEGUNDO PARCIAL } \\
\hline & $n^{\circ}$ & $\%$ & $\mathrm{n}^{\circ}$ & $\%$ \\
\hline Alumnos presentados & 46 & & 44 & \\
\hline Suspensos & 24 & $52,17 \%$ & 1 & $2,27 \%$ \\
\hline Aprobados & 19 & $41,30 \%$ & 30 & $68,18 \%$ \\
\hline Notables & 3 & $6,52 \%$ & 11 & $25,00 \%$ \\
\hline Sobresalientes & 0 & $0,00 \%$ & 2 & $4,55 \%$ \\
\hline Total & 46 & $100,00 \%$ & 44 & $100,00 \%$ \\
\hline \multirow[t]{2}{*}{ GRUPO sin Twitter } & \multicolumn{2}{|c|}{ PRIMER PARCIAL } & \multicolumn{2}{|c|}{ SEGUNDO PARCIAL } \\
\hline & $n^{\circ}$ & $\%$ & $n^{\circ}$ & $\%$ \\
\hline Alumnos presentados & 96 & & 92 & \\
\hline Suspensos & 34 & $35,42 \%$ & 10 & $10,87 \%$ \\
\hline Aprobados & 39 & $40,63 \%$ & 42 & $45,65 \%$ \\
\hline Notables & 22 & $22,92 \%$ & 29 & $31,52 \%$ \\
\hline Sobresalientes & 1 & $1,04 \%$ & 11 & $11,96 \%$ \\
\hline Total & 96 & $100,00 \%$ & 92 & $100,00 \%$ \\
\hline
\end{tabular}

Como se puede observar en la Tabla 5, los resultados del grupo mejoran significativamente tras el uso de Twitter como herramienta docente, disminuyendo el porcentaje de suspensos al 2,27\%, cuando se situaba en más del $50 \%$ anteriormente a la experiencia. Además, también mejoran las calificaciones de los alumnos y con ello la nota media del grupo. En el primer parcial, no había ningún sobresaliente y sólo 3 notables. Con el uso de Twitter, 11 alumnos obtienen una calificación de notable y 2 de sobresaliente.

En el otro grupo, ya que en el primer parcial obtuvo un buen porcentaje de aprobados, 64,58\%, no se realizó la experiencia de Twitter. No obstante, pese a no haber implementado la experiencia de Twitter dicho grupo también mejora sus resultados finales, incrementado el porcentaje de aprobados hasta un $89 \%$ en el segundo parcial. Esta evolución positiva viene explicada por el buen nivel académico del grupo y por el carácter anual de la asignatura, que hace que el alumno que ya había superado un parcial se prepare la materia con mayor compromiso o bien hace que el alumno que había suspendido el primer parcial estudie con mayor interés la materia para evitar, en ambos casos, ir a la convocatoria final para examinarse de la totalidad del temario.

Complementariamente a estos resultados académicos se consiguieron otros de índole emocional y personal. Se consiguió un alto nivel de motivación y satisfacción del alumnado, se creó una fuerte sensación de grupo entre todos ellos, hasta el punto de que se sentían identificados bajo el nombre del hashtag. A partir de aquí surgen sinergias, se crea un nuevo vínculo, ya que deciden hacer un grupo de whatsapp, al que llaman, también, "\#ideasfinanzas", para estar permanentemente conectados y ayudarse en preparar no sólo nuestra asignatura sino todas las demás. En este grupo, la profesora se mantiene al margen, no forma parte de él. Sobre la base de sus comentarios, esta experiencia también fue positiva en los resultados académicos que consiguieron en otras asignaturas, porque, en sus palabras, "este grupo que se había originado hacía que siempre se sintiesen acompañados y encontraron en ellos una fuente de motivación".

Actualmente, el hashtag sigue activo y muchos de estos alumnos siguen unidos y manteniendo el contacto y las relaciones personales que surgieron. Lo cual nos sugiere 
que el uso de esta red social mejora las relaciones personales en la clase, y también podría ser una vía para incrementar la satisfacción del alumnado.

\section{CONCLUSIONES}

La implementación de nuevas tecnologías de la información y las comunicaciones (TICS) aplicadas al ámbito docente está teniendo una repercusión e importancia cada vez más relevante en la educación universitaria. Bajo este marco, en este trabajo se ha realizado un experimento de campo cuyo objetivo es analizar si se produce una mejora en la participación activa y los resultados académicos del alumnado al incorporar tecnologías de la información y las comunicaciones (TICs) como base de las metodologías docentes. Para ello, se han utilizado los resultados obtenidos en tres asignaturas diferentes del área financiera durante un curso académico (2011/2012). Las TICs utilizadas en nuestro experimento de campo fueron la plataforma virtual (WebCT) y la red social Twitter.

Los resultados obtenidos pueden dividirse en dos partes, uno por cada una de las dos hipótesis establecidas. En relación a la primera hipótesis a testar lla participación activa del alumnado, medida a través del uso intensivo de la plataforma virtual, mejora los resultados académicos), nuestras evidencias empíricas muestran que existe una clara relación entre la participación activa en WebCT durante el curso y el resultado conseguido en el examen. Esto significa que fomentar una participación activa por medio de WebCT y el esfuerzo hecho por los alumnos de utilizar de forma intensiva esta herramienta tiene consecuencias positivas sobre los resultados académicos.

En relación con la segunda hipótesis a testar, (las redes sociales como instrumento de evaluación continua mejoran los resultados académicos), nuestros resultados muestran que el uso de Twitter como herramienta docente decrementó el porcentaje de alumnos suspensos de forma muy significativa, pasando de más del $50 \%$ al 2,27\%. Además de los alumnos que superan la asignatura, el uso de esta red social mejoró las calificaciones de los alumnos y con ello la nota media del grupo. En el primer parcial, no había ningún sobresaliente y sólo 3 notables. Con el uso de Twitter, 11 alumnos obtuvieron una calificación de notable y 2 alumnos de sobresaliente. Finalmente, además de los resultados académicos, Twitter incrementó la motivación y satisfacción del alumnado ya que aumentó las relaciones personales entre los miembros de la clase y la sensación de unión del grupo. Todo ello, redunda en una mejor atención, trabajo y dinamismo de la clase.

\section{BIBLIOGRAFÍA}

AlCÁNTARA, A. [2012]: I Informe de Infoempleo y Adecco sobre redes sociales y mercados de trabajo. Disponible en http://yoriento.com/2013/01/comoencuentran-las-empresas-a-los-profesionales-que-buscan-trabajo-12-datossobre-reclutamiento-y-busqueda-de-empleo-761.html/

Alfalla-LUQUe, R., MedinA-LóPez, C., ARenAS-MÁRQUez, F.J. [2011]: Mejorando la formación en Dirección de Operaciones: La visión del estudiante y su respuesta ante diferentes metodologías docentes. Cuadernos de Economía y Dirección de la Empresa, Vol. XIV, núm. 1, pp. 40-52.

ARbaUGH, J.B., DeSAl, A., RAU, B., SRIDHAR, B.S. [2010]: "A review of research on online and blended learning in the management disciplines: 1994-2009". Organization Management Journal, Vol. 7, núm. 1, pp. 39-55.

Catenazzl, N., SOmmaruga, L. [1999]: The evaluation of the Hyper Apuntes interactive learning environment. Computers and Education, Vol. XXXII, núm. 1, pp. 35-49. 
Collaud, G., Gurtner, J.L., Coen, P.F. [2000]: Design and use of a hypermedia system at the University level. Journal of Computer Assisted Learning, núm. 16, pp. 137147.

Garandillas Iglesias, M., Somohano Rodríguez, F.M.; Martínez García, F.J. [2011]: Innovación y evaluación en el marco del Espacio Europeo de Educación Superior: una experiencia piloto utilizando herramientas Web 2.0. Educade, Revista de Educación en Contabilidad, Finanzas y Administración de Empresas, núm. 2, pp. 85-111.

KULL, J.L., MACKINNON, D.P. [2001]: Multilevel modeling of individual and group level mediated effects. Multivariate Behavioral Research, Vol. 36, No. 2, pp. 249-277.

LeEDS, M., StULL, W., WeSTBROOK, J. [1998]: Do changes in classroom techniques matter? Teaching strategies and their effects on teaching evaluations. Journal of Education for Business, Vol. LXXIV, núm. 2, pp. 75-78.

LÓPEZ-PÉREZ, M.V., PÉrEZ-LÓPEZ, M.C.; ROdRíGUeZ-ARIZA, L. [2011]: "Blended learning in higher education: Students' perceptions and their relation to outcomes". Computers \& Education, Vol. 56, núm. 1, pp. 818-826.

Mateos-Aparicio, M. [2012]: Sitios de redes sociales y mediación en procesos de enseñanza-aprendizaje, http://ddd.uab.cat/pub/dim/dim_a2012m1n22/dim_a2012m1n22a5.pdf

MAYOR, C. [1997]: La supervisión clínica como estrategia de asesoramiento. En Asesoramiento curricular y organizativo en educación, Marcelo García, C. y López Yañez, J. (coord.). Ed. Ariel.

Miguel-DÁVILA, J.A., LÓPEZ-BerzosA, D., MARTín-SÁnCheZ, M. [2011]: ¿Una participación activa del alumno pronostica una buena nota en el examen? Working Papers on Operations Management. Vol. III, núm 2, pp. 71-83.

Oltra Mestre, M.J., García Palao, C., Flor Peris, M.L., Boronat Navarro, M. [2011]: Aprendizaje activo y desempeño del estudiante: diseño de un curso de dirección de la producción. Working Papers on Operations Management. Vol. III, núm 2, pp. 84-102.

PREACHER, K.J., RUCKER, D.D., HAYES, A.F. [2007]: Addressing moderated mediation hypotheses: Theory, methods, and prescriptions. Multivariate Behavioral Research, Vol. 42, No. 1, pp. 185-227.

Rayburn, L.G., Rayburn, J.M. [1999]: Impact of course length and homework assignments on student performance. Journal of Education for Business, Vol. LXXIV, núm. 6, pp. 325-331.

ROMERO FRÍAS, E. [2010]: El empleo de wikis en la docencia universitaria: resultados de una experiencia en contabilidad. Educade, Revista de Educación en Contabilidad, Finanzas y Administración de Empresas, núm 1, pp. 43-58.

RuIz, J. [1992]: Gerencia en el aula. Yaracuy, Venezuela :INSTIVOC.

Sacristan-Diaz, M., Garrido-Vega, P., Gonzalez-Zamora, M., Alfalla-luque, R. [2012]: "Impacts of Teaching and Assessment Methods on Academic Performance in an OM Course. Ponencia en Congreso. 4th World Conference POMS-EurOMA. Amsterdam.

SÁNCHeZ MORENO, M. [2008]: "Asesoramiento en la Universidad. Poniendo a trabajar a la Experiencia". Profesorado. Revista de curriculum y formación del profesorado.

SÁNCHEZ MORENO, M.; MAYOR RUIZ, C. [2006]: "Los jóvenes profesores universitarios y su formación pedagógica. Claves y Controversias". Revista de Educación, Vol. 339, pp. 923-946.

SARRAMONA, J. [1988]: COMUNICACION Y EDUCACION, eds. CEAC, S.A., Barcelona.

TANG, T.L.; AUSTIN, M.J. [2009]: "Students' perceptions of teaching technologies, application of technologies, and academic performance". Computers \& Education, Vol. 53, núm. 4, pp. 1241-1255.

TAPIA, J.A. [1991]: Motivación y aprendizaje en el aula. Cómo enseñar a pensar, ed. Santillana, Madrid, 1991. 
Valentín, A., Mateos, P.M., González-Tablas, M.M., Pérez, L., López, E. García, I. [2013]: "Motivation and learning strategies in the use of ICTs among university students". Computers \& Education, Vol. 61, 52-58.

WANG, Q. [2008]: "A generic model for guiding the integration of ICT into teaching and learning". Innovations in Education and Teaching International, Vol. 45, núm. 44, pp. 411-419.

YOURSTONE, S.A., KRAYE, H.S., AlbaUM, G. [2008]: "Classroom Questioning with Immediate Electronic Response: Do Clickers Improve Learning?" Decision Sciences Journal of Innovative Education, Vol. 6, núm. 1, pp. 75-88. 